\title{
REBCO tape performance under high magnetic field ${ }^{\star}$
}

\author{
Tara Benkel ${ }^{1,2, a}$, Yasuyuki Miyoshi ${ }^{2}$, Xavier Chaud $^{2}$, Arnaud Badel ${ }^{1}$, and Pascal Tixador ${ }^{1}$ \\ ${ }^{1}$ G2Elab/Institut Néel, CNRS, 38042 Grenoble, France \\ ${ }^{2}$ LNCMI, CNRS, 38042 Grenoble, France
}

Received: 23 November 2016 / Received in final form: 15 April 2017 / Accepted: 9 June 2017 (c) EDP Sciences 2017

\begin{abstract}
New improvements in high temperature superconductors (HTS) make them a promising candidate for building the next generation of high field magnets. As the conductors became recently available in long length, new projects such as NOUGAT (new magnet generation to generate Tesla at low cost) were started. This project aims at designing and building an HTS magnet prototype generating $10 \mathrm{~T}$ inside a $20 \mathrm{~T}$ resistive magnet. In this configuration, severe mechanical stress is applied on the insert and its extremities are subject to a high transverse component of the field. Because the conductor has anisotropic properties, it has to be studied carefully under similar conditions as the final prototype. First, this paper presents both the NOUGAT project and its context. Then, it shows the experimental results on short HTS tapes studied under high magnetic field up to $23 \mathrm{~T}$ with varying orientation. These results allow validating the current margin of the prototype. Finally, a first wound prototype is presented with experimental results up to 200 A under $16 \mathrm{~T}$.
\end{abstract}

\section{Introduction}

The national high field magnet laboratory (LNCMI) in Grenoble allows researchers from all around the world to work under high magnetic field up to $36 \mathrm{~T}$. In order to achieve this field strength resistive electromagnets are necessary. However, this implies large energy consumption, up to $24 \mathrm{MW}$ in the case of LNCMI. Such facilities are rare due to their complexity and high expenses. Moreover, the high energy dissipation requires an efficient water cooling system, which represents in the case of LNCMI, $300 \mathrm{~L} / \mathrm{s}$.

The constant increase of electricity prices and the pursuit of experiments under higher magnetic fields limit the use of traditional conductors. Superconductors on the other hand have exceptional properties under low temperature $[1,2]$, where they do not show any resistance against the current passing through them.

There are two types of superconductors. The first discovered is called low temperature superconductors (LTS) and they have been studied extensively. They are already used in industrial application such as magnetic resonance imaging (MRI), or large hadron collider (LHC) magnets at CERN. Nevertheless, they seem to have reached their limit in terms of magnetic field with the Bruker NMR magnet record of $23.5 \mathrm{~T}$ [3].

The second type of superconductors is the main focus of this paper, called high temperature superconductors

\footnotetext{
${ }^{a}$ e-mail: tara.benkel@lncmi.cnrs.fr

${ }^{\star}$ Contribution to the topical issue "Electrical Engineering Symposium (SGE 2016)", edited by Adel Razek
}

(HTS). As their properties are continuously improved, the studies continue to build high field magnets. HTS tapes can carry high current densities even at field strengths of $30 \mathrm{~T}$ and thanks to the Hastelloy substrate, severe mechanical stresses are tolerable. Recently available in long lengths, they are now studied in several research projects, especially for high field magnet applications.

The National High Magnetic Field Laboratory of Tallahassee has designed and built a full-superconducting magnet to provide $32 \mathrm{~T}$ in its center. The outer LTS magnet generates $15 \mathrm{~T}$ and the HTS insert made of two coils generates $17 \mathrm{~T}$ [4]. A second magnet project at Tohoku University, Japan aims at delivering a fullsuperconducting magnet, expected to generate $20 \mathrm{~T}$ [5] with an 11.5 T HTS insert. A third superconducting magnet is under study at the Chinese Academy of Sciences [6]. The HTS insert of this magnet is designed to generate 7.5 $\mathrm{T}$ in its center. The Francis Bitter Magnet Laboratory (FBML) in Boston is working on an HTS insert of 18.8 T expected to work inside a NMR LTS $11.7 \mathrm{~T}$ magnet already existing at the FBML [7].

The ongoing projects in Grenoble include NOUGAT (new magnet generation to generate Tesla at low cost). It aims at building an HTS insert generating $10 \mathrm{~T}$ in its center, which is intended to work inside a $20 \mathrm{~T}$ resistive magnet already existing at LNCMI, in order to generate a total of $30 \mathrm{~T}$ [8]. The main goal is not to reach higher field than the already existing magnets, but to show the possibility of using HTS conductors to build a new generation of high field magnets. Moreover, the use of a resistive outer magnet allows working only with the HTS 
insert, avoiding all the issues due to coupling effects with a LTS background field.

This study adds substantial new information to the field of applied superconductivity. In a near future, HTS are expected to bring many improvements to electrical engineering such as energy storage, transport and power electronics [9].

\section{Context}

\subsection{REBCO tapes}

This project studies rare earth barium copper oxide called REBCO HTS tapes. The most well-known rare earth used for REBCO is yttrium because YBCO was the first to be discovered with a critical temperature above $77 \mathrm{~K}$, the liquid nitrogen temperature. This promised a considerable decrease in the cost of superconducting applications.

Above a given temperature, superconductors quench; this means that the material is back to its resistive state. This temperature is called the critical temperature of the superconductors. The tape studied in the present paper is made of yttrium but some others are also sold, made with different materials such as gadolinium for example. Similar results are obtained with them.

Second generation superconductors are for now only sold in the shape of a tape with a thickness of $0.1-0.2 \mathrm{~mm}$, width of $4-12 \mathrm{~mm}$ and are made of a stack of layers as shown in Figure 1 [10]. The superconducting layer thickness is about 1 or $2 \mu \mathrm{m}$, making up only $1 \%$ of the total thickness. The critical radius of curvature is limited by the superconducting layer. It is approximately $6 \mathrm{~mm}$, meaning that if the tape is bent beyond this point, the superconducting layer will be permanently damaged.

The characteristics of the tape studied in this paper are summarized in the following table (Tab. 1).

High temperature superconductors can carry high currents even under high magnetic field, especially at low temperature. Nevertheless, the critical current decreases with increasing field. It is also highly linked to the orientation of the tape with respect to the field [11]. It is not uncommon that the amount of current carried under transverse field is 1.5-4 times lower than under the same field amplitude but parallel to the tape. This anisotropy has to be taken into consideration when dimensioning the magnet [12]. Eventually, HTS tapes can be subject to severe mechanical stress thanks to their substrate, made of Hastelloy ${ }^{\circledR}$, a high performance nickel tungsten alloy.

For these reasons, HTS are studied in all the projects presented above and the NOUGAT project.

\subsection{NOUGAT project}

\subsubsection{Building constraints}

As the conductor is sold in the shape of a tape, coils are laterally wound, called pancakes. If two of them are wound together with the same length of tape, it is called double

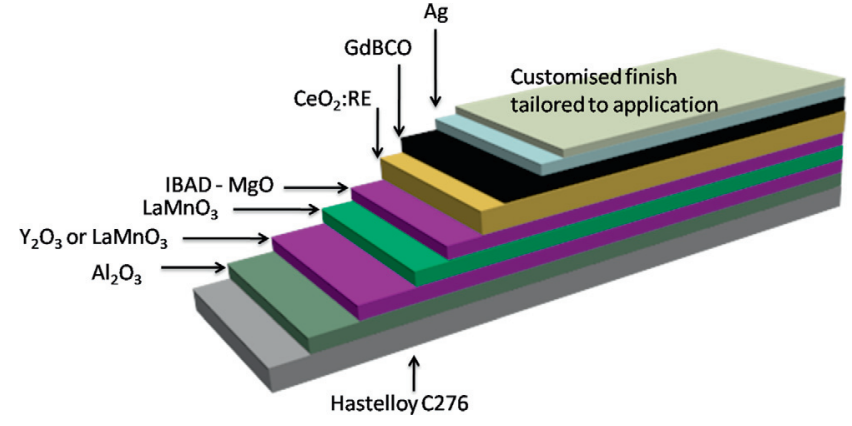

Fig. 1. Example of a HTS tape cross section, according to SuperOx ${ }^{\circledR}$ conductor [10], illustration is not to scale.

Table 1. Details of SuperOx ${ }^{\circledR}$ REBCO tape.

\begin{tabular}{lc}
\hline Characteristics & \\
\hline Width & $4 \mathrm{~mm}$ \\
Total thickness & $\sim 120 \mu \mathrm{m}$ \\
Substrate layer & $60 \mu \mathrm{m}$ \\
Silver layer & $\sim 2 \mu \mathrm{m}$ \\
Copper $(\times 2)$ layer & $20 \mu \mathrm{m}$ \\
Deposited polyimide & $10 \mu \mathrm{m}$ \\
Superconducting layer & $2 \mu \mathrm{m}$ \\
$I_{\mathrm{c}}$ min $(77 \mathrm{~K}$, self-field $)$ & $120 \mathrm{~A}$ \\
\hline
\end{tabular}

pancake. In the NOUGAT project, the insert is designed as a stack of pancakes or double pancakes.

The insert geometry is mostly constrained by the already existing resistive outer magnet. For example, the outer diameter of the insert has to be equal to or smaller than $110 \mathrm{~mm}$. Furthermore, existing experimental setups must fit inside the HTS insert. This puts a constraint on the internal bore diameter, which was chosen to be $50 \mathrm{~mm}$. In this configuration, the maximum height is about $150 \mathrm{~mm}$ to keep the insert in the background magnetic field which is homogenous. This height allows building 18 double pancakes wound with a 4-mm width tape. The average thickness of the conductor is about $0.135 \mathrm{~mm}$. In this configuration, each pancake is made of 185 turns. The insert model is summarized in Table 2.

Two solutions are considered for building the pancake coil. The first solution is to use an electrical insulated conductor so each turn of the pancake is electrically isolated from the next one. The second solution is to use a non-electrically isolated HTS tape and to co-wind this conductor with a resistive metallic layer like steel for example [13].

This latter technique is used to protect the coil in case of a partial quench of the conductor as it is one of the main issues of HTS windings. It allows thermal stability $[14,15]$. This technique is used for the NOUGAT project in order to do non destructive tests on windings. Nevertheless, even if this process increases the coil stability, it also has some negative effects. It increases the charge and discharge time of the coil and also reduces the homogeneity and stability of the generated field. 
Table 2. Summary of the model.

\begin{tabular}{lc}
\hline Characteristics & \\
\hline Outer diameter & $110 \mathrm{~mm}$ \\
Inner diameter & $50 \mathrm{~mm}$ \\
Height & $150 \mathrm{~mm}$ \\
Double pancake numbers & 18 \\
Conductor width & $4 \mathrm{~mm}$ \\
Turns number/Pancake & 185 \\
\hline
\end{tabular}

Hence, we also work on insulated coils that have better field linearity [16] and lower time constants. This is a main advantage when the background is made with LTS since, in the event of a quench, the HTS insert has to be discharged quickly to avoid high currents due to coupling [13]. In this paper, the electrically insulated coil is presented.

The geometry of the insert is one of the main constraints. In order to reach the project goal, the insulation had to be chosen as thin as possible. Deposited polyimide was selected rather than insulating tape wound around the superconducting tape. In the first case, the layer will have a thickness of about $10 \mu \mathrm{m}$ then between $25 \mu \mathrm{m}$ and $50 \mu \mathrm{m}$ for the second case [17]. The present study has been realized on a conductor provided by Superox ${ }^{\circledR}$ The characteristics of the tape are summarized in Table 1 (Sect. 2.1).

\subsubsection{Magnetic field distribution and consequences}

The total magnetic field distribution is shown on Figure 2. It is the result of the addition of the magnetic field generated by the HTS insert (10 T in its center) and the resistive magnetic field background (20 $\mathrm{T}$ in its center). The resistive outer magnet is shown on the right of the figure, delimited by the black tilled line. The magnetic field generated by this latter magnet is both measured and calculated by LNCMI. The magnetic field generated by the HTS insert (black line) is calculated thanks to the FLUX $^{\circledR}$ software, G2Elab/Cedrat [18]. The addition is also evaluated thanks to the same software. The magnetic field in the center of the total fields generated by the HTS and by the resistive magnet is $30 \mathrm{~T}$.

In this configuration, the maximum transverse component of the field is at the edges of the windings, as expected [12]. It reaches a maximum of $3.5 \mathrm{~T}$ for a field modulus of about $20 \mathrm{~T}$. Hence, in this area, the magnetic field is no longer parallel to the surface of the HTS tape. In the following, $0^{\circ}$ angle is used to refer to a magnetic field parallel to the tape surface and $90^{\circ}$ is used to refer to a magnetic field perpendicular to the conductor surface. Here, in the area of the highest transverse field, the magnetic field direction and the surface of the tape create an angle of about $14^{\circ}$. This angle is called the incident angle.

The winding has to be able to carry $200 \mathrm{~A}$ in order to reach $10 \mathrm{~T}$ in its center and fill the geometric constraints of the project. In this configuration, $200 \mathrm{~A}$ equal

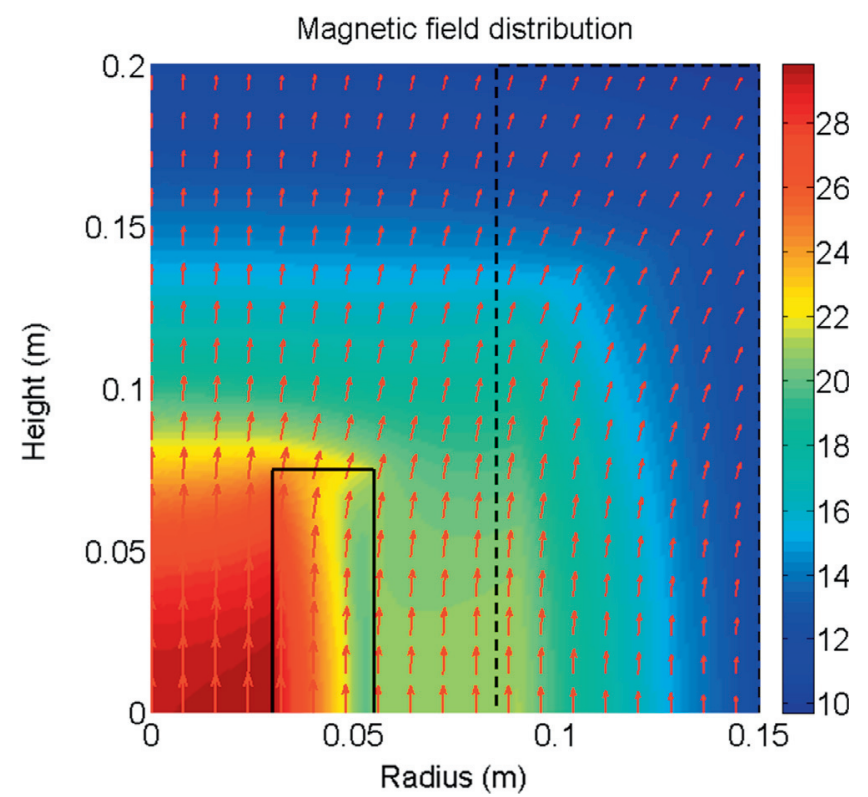

Fig. 2. Magnetic field distribution (cross section) in the assembly of the $10 \mathrm{~T}$ HTS insert inside the $20 \mathrm{~T}$ resistive outer magnet. Graphics drawn thanks to the FLUX ${ }^{\circledR}$ software.

to a current density of $370 \mathrm{~A} / \mathrm{mm}^{2}$ passing through the conductor. This value has to be low enough to respect the current margin in order to prevent the coil from quenching and then damaging the winding.

The conductor has to be studied under similar conditions as the final prototype working conditions. This means under the same value of the magnetic field intensity and orientation. The conductor should be studied between $18 \mathrm{~T}$ and $30 \mathrm{~T}$ with a field orientation from $0^{\circ}$ to $15^{\circ}$. In order to obtain the full trends of the curves, the tape will be also studied under $18 \mathrm{~T}$ and with an orientation from $0^{\circ}$ to $90^{\circ}$.

\section{Experimental workbench for short tape samples}

The HTS tapes have to be studied under conditions as similar to the final prototype in order to validate the project expectations.

\subsection{Experimental setup and measurement probe}

Critical current measurements of several different tapes under various high magnetic fields can be found in the literature but they are mostly measured under specific magnetic field orientation, especially under parallel and perpendicular fields [19]. Some other orientations between these two specific cases can be also found but most of the time, the width of the tape is reduced in order to accommodate the measurement probe limitation [20,21]. At $4.2 \mathrm{~K}$ (liquid helium bath), more than $1000 \mathrm{~A}$ can pass through a 4 -mm tape in parallel magnetic field [22]. 
(a)

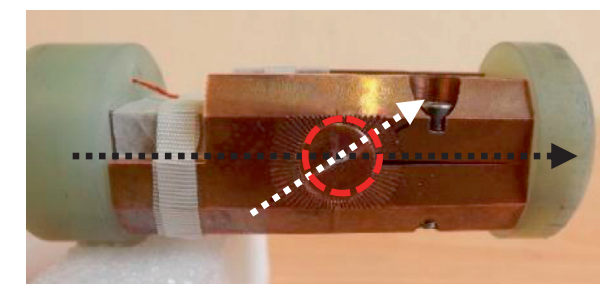

(b)

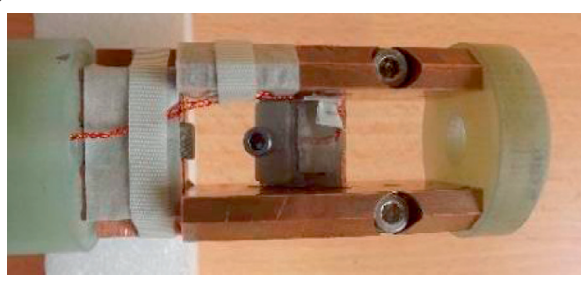

(c)

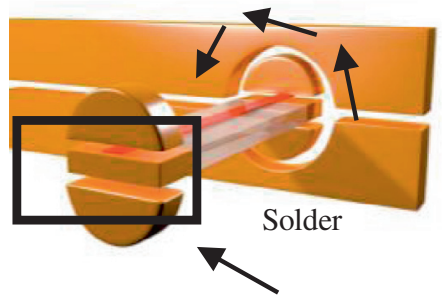

Fig. 3. (a) Measurement probe extremity, view from the side. (b) Measurement probe extremity, view from the top. (c) Draft of the extremity of the measurement probe, view from the side.

The free area under magnetic field is narrow so measurement probes cannot easily carry enough current even at low temperature. Nevertheless, the fact that the tape is cut in order to reduce its width can be responsible for some defects in the conductor leading to a decrease in performance [23].

In LNCMI, one of the measurement probes has been designed in order to carry $2000 \mathrm{~A}$ under $4.2 \mathrm{~K}$ and to study the angular dependency of short straight HTS samples. The sample is located at the extremity of the measurement probe shown in photographs (a) and (b) on Figure 3. The draft in (c) shows how the YBCO sample is setup (red) on the rotating part of the measurement probe. The copper pieces are opaque orange and the glass fiber is transparent white.

The straight samples are prepared to measure about $30 \mathrm{~mm}$. The measurement probe allows studying the sample from $0^{\circ}$ to $360^{\circ}$ with an angular step of $2.5^{\circ}$ under magnetic field, with accuracy of $1^{\circ}$. Voltage taps are soldered on the centre of the tape at $1 \mathrm{~cm}$ from each other. The voltage threshold $E_{\mathrm{c}}$ is setup to detect the critical current at $1 \mu \mathrm{V} / \mathrm{cm}$.

The thermal behavior of the measurement probe is monitored using Cernox ${ }^{\circledR}$ sensors. The voltages along the current leads are recorded using voltage taps. The measurement probe allows working at different temperatures achieved by a heater plate that can be added at the extremity of the probe. Nevertheless, the following results are given in liquid helium bath, $4.2 \mathrm{~K}$.

\subsection{Results}

\subsubsection{Results in detail}

In this paper $4 \mathrm{~mm}$ wide tape was used from SuperOx ${ }^{\circledR}$. As shown in Table 1 (Sect. 2.1).

Initially, the conductor's critical current is tested in fields up to $12 \mathrm{~T}$. Then it is studied until $23 \mathrm{~T}$. For both

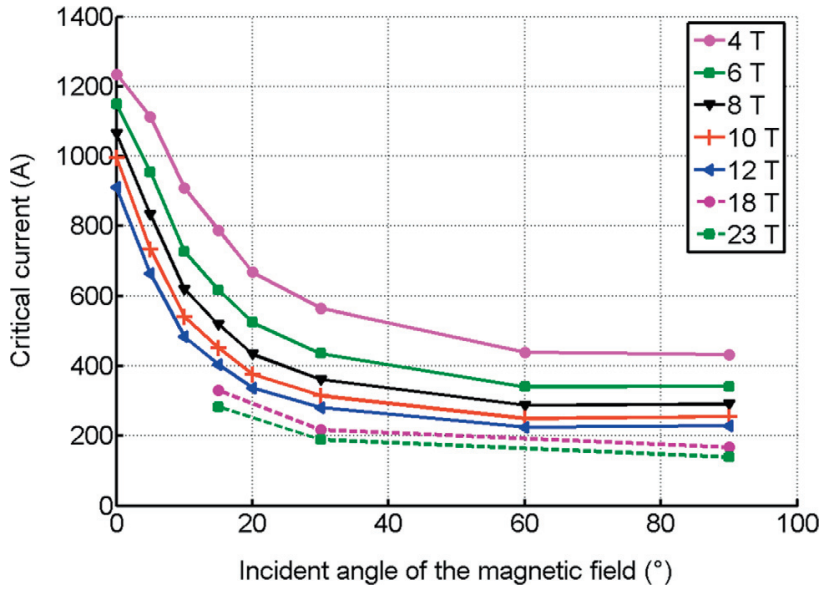

Fig. 4. Critical current value according to the intensity and the orientation of the background magnetic field applied on the tape.

cases, the conductor is studied from $0^{\circ}$ (parallel field) to $90^{\circ}$ (perpendicular field) on the following specific intermediate values : $5^{\circ}, 10^{\circ}, 15^{\circ}, 30^{\circ}$ and $60^{\circ}$. Results are presented on the following graph (Fig. 4). The critical current measurements are given according to the incident angle for several magnetic field values.

The critical current values decrease by $30 \%$ for a sample rotated from $0^{\circ}$ to $15^{\circ}$. On the opposite, current performance does not really increase anymore for incident angles higher than $50^{\circ}$. However, according to Section 2.2, only small angles are interesting for the HTS inserts. Hence, the decrease of $30 \%$ is a major concern to evaluate the critical current that will limit the performance of the coil [4].

Nevertheless, these measurements, especially at low angles, are difficult to realize. One can see that results are missing in the graph for incident angles lower than $15^{\circ}$ and magnetic field higher than $12 \mathrm{~T}$. In this configuration, the current passing through the tape combined with 


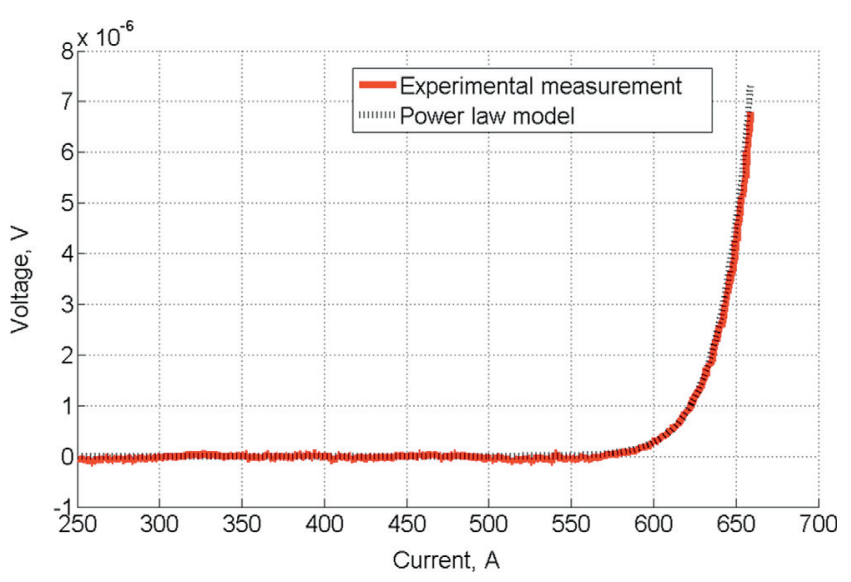

Fig. 5. Quench of REBCO tape from its superconducting to its dissipative state under $8 \mathrm{~T}$ with an incident angle of $10^{\circ}$. Both experimental curve and numerical curve are shown.

the magnetic field applied on it creates strong mechanical stresses. Under these conditions, the conductor can be easily damaged. For these reasons, the samples have to be changed for a few measurements, especially at low angles. The data shown in the graph are the average of all the measurements obtained for the same tests conditions. Missing points have been numerically extrapolated (Sect. 4.1).

According to the magnetic field distribution given in Figure 2, the HTS insert is expected to work under magnetic field up to $30 \mathrm{~T}$. At the time of the experiment, only fields up to $23 \mathrm{~T}$ were available so that the missing points have to be numerically extrapolated up to $30 \mathrm{~T}$. All the measurements and the extrapolations are developed in Section 4.1.

\subsection{2 n Calculation for the power law}

The power law (1) is commonly used to model the $E(J)$ behavior in superconductors.

$$
E(J)=E_{\mathrm{c}}\left(\frac{J}{J_{\mathrm{c}}}\right)^{n},
$$

$E$ is the electric field in $\mathrm{V} / \mathrm{m} . E_{\mathrm{c}}$ is the same as defined, i.e. $1 \mu \mathrm{V} / \mathrm{cm} . J$ is the current density and $J_{\mathrm{c}}$ is the critical current density. Both of them are in $\mathrm{A} / \mathrm{mm}^{2}$. $n$ has no unit and it is the superscript of the law, called the power factor. Figure 5 shows a comparison between the experimentally and numerically obtained $E(J)$ curves. The power law constants, $J_{\mathrm{c}}$ and $n$ are derived from experiments.

Figure 6 shows the $n$ values estimation extrapolated from the experiments, Section 3.2.1.

If the power factor is close to $0 \mathrm{~T}$, the $n$ value is high. Then, it decreases until $4 \mathrm{~T}$ and stays approximately stable until $12 \mathrm{~T}$. It also seems to decrease with the increase of the angle between the direction of the magnetic field and the surface of the tape. The $n$ calculation has consequences in the model of the superconducting behavior. In the present work, the $n$ values are similar to the ones found in literature [24].

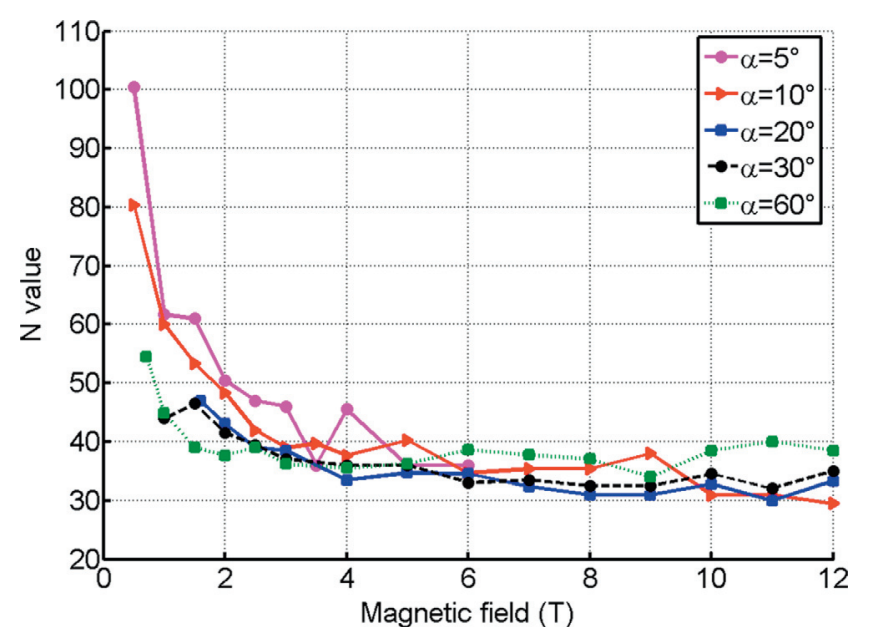

Fig. 6. Power factor values according to the magnetic field intensity and orientation.

\section{Results}

The limited availability of facilities and the difficulty to set up a reliable sample made measurements impossible to realize above $23 \mathrm{~T}$. Nevertheless, the final prototype is expected to work with $30 \mathrm{~T}$ in its center so the previous results need to be extrapolated in order to evaluate the current margin.

\subsection{Results extrapolation}

Kim's model [25] is used to extrapolate the previous results, using the following formula (2).

$$
J_{\mathrm{c}}(B)=\frac{J_{\mathrm{c}}(0)}{1+\frac{|B|}{B_{0}}},
$$

$J_{\mathrm{c}}(0)$ is the hypothetic value of $J_{\mathrm{c}}$ in self-field. As the critical current is huge for low field, it is not possible anymore to neglect the magnetic field generated by the tape itself. The critical current without any background field is about $1450 \mathrm{~A}$ for the tape studied in this paper. $J_{\mathrm{c}}$ is then numerically approximated. Above the $B_{0}$ value, the critical current starts to saturate slightly [26]. The results obtained with the Kim's model on the experimental results presented in Section 3.2.1 are shown in Figure 7. Constants are derived from experimental results. The error is less than $4 \%$ for incident angles lower than $20^{\circ}$ and above $10 \%$ for the incident angles higher than $20^{\circ}$. One can see that the numerical model does not fit the experimental curves trend for magnetic field lower than $10 \mathrm{~T}$ with incident angles higher than $20^{\circ}$.

Nevertheless, only critical current values for low incident angles and high magnetic field are interesting for the insert design so the model is sufficient for the project.

\subsection{Margins calculation}

Kim's model makes it possible to evaluate the limiting critical current of the full insert. The critical current can 


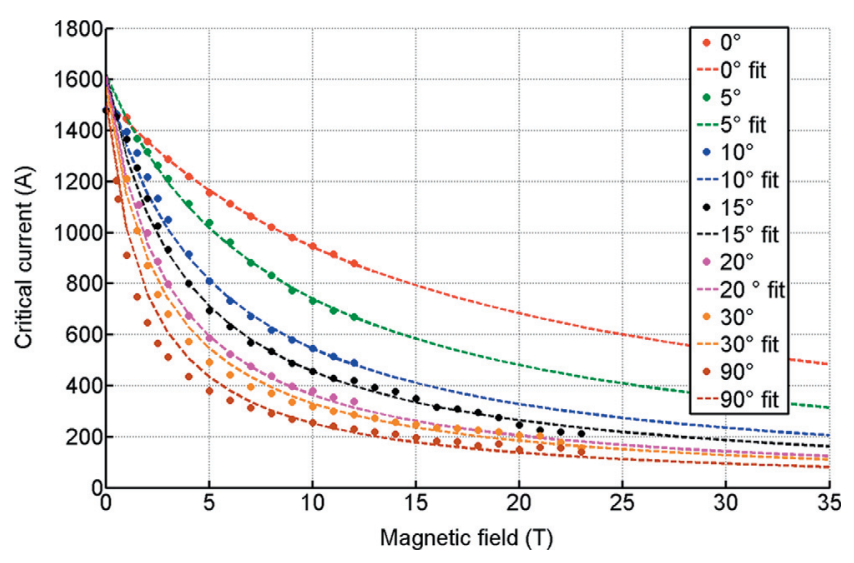

Fig. 7. Critical current values (dots) for various magnetic field orientation and intensity compared to the Kim's model (tilled lines).

be evaluated on the whole insert section according to the experimental results on short length and the magnetic field distribution presented in Section 2.2. Hence, the critical current distribution can be evaluated on the cross section of the insert according the magnetic field intensity and orientation. The critical current distribution on the cross section is shown in Figure 8a. The limiting critical current is about $350 \mathrm{~A}$. It is situated at the edges of the winding as expected (Sect. 2.2). As the calculated nominal current is $200 \mathrm{~A}$, the final prototype is expected to work at $57 \%$ of its limiting critical current. In this case, the critical current is different than the limiting critical current because only the critical current values on a specific area of the winding are considered [4].

The nominal current is validated. This value can then be used to calculate the mechanical radial stress $\sigma$ applied on the winding. A pessimist hypothesis is taken in which the turns are considered as if they were acting independently [27]. The following formula (3) is used.

$$
\sigma=J \times B_{z} \times r
$$

$R$ is the winding radius and $J$ is the nominal current density. The mechanical stress distribution on the cross section of the insert is shown in Figure 8b. The maximum of the mechanical stress is $420 \mathrm{MPa}$ and is applied on the outer turns of the winding.

One can see that calculated and measured mechanical stresses are similar in [28].

\section{First wound prototypes}

After studying short tape samples, several wound prototypes are built. The first one is a double pancake wound with the same 4-mm width conductor provided by SuperOx ${ }^{\circledR}$ studied in short length. This test aims at studying the winding process given the weakness of the tape (Sect. 2.1). One of the other main goals is to appreciate the feasibility of building all the wound parts of the final prototype.
The main objective of this first prototype is to show that $200 \mathrm{~A}$ can be passed through the coil under $20 \mathrm{~T}$ both on the tape parallel to the field but also on the critical area of the winding like the edges of the double pancake. This coil is expected to be moved in the height of the background field in order to recreate the similar constraints as the one of the final prototype in nominal working conditions.

\subsection{Double pancake and winding process}

As explained above, the double pancake is wound with an electrically insulated tape, taken from the same length as the short samples shown above. The coil characteristics are presented in Table 3.

The double pancake assembly is shown in Figure 9a before being wrapped. It is a double pancake without a soldered joint in the middle of the winding, the two pancakes are wound with the same conductor length. The middle of the length is first fixed on the mandrel. Then half of the tape stays on a satellite fixed on the mandrel while the other half is wound on the mandrel, Figure $9 \mathrm{~b}$. Hence, the satellite follows the rotation while winding the first pancake.

The mandrel is made of glass fiber and covers a copper mandrel (A). The first tape extremity is clamped on the copper cylinder in the bottom of the assembly $(\mathrm{E})$. This cylinder is tight on the copper mandrel (A). It allows the current to reach the top copper ring (C) and then the current lead (B). The other tape extremity of the winding is soldered on the copper cylinder $(\mathrm{F})$ in the middle of the assembly. Then the current can reach the second current lead (D).

Eventually, the two last turns are soldered on themselves for mechanical reinforcement and to relieve a part of the mechanical stress. Finally the winding is wrapped in a protective coating made of epoxy glass fibers. This assembly is then mounted on a measurement probe. Current of 2000 A can be passed through the probe at $4.2 \mathrm{~K}[20]$.

\subsection{Experimental results}

The double pancake was tested in liquid helium bath, at $4.2 \mathrm{~K}$, with a constant current of $200 \mathrm{~A}$ in a background field from $0 \mathrm{~T}$ to $16 \mathrm{~T}$. During the $200 \mathrm{~A} 16 \mathrm{~T}$ test, the soldered current lead burnt. Neither voltage appearance nor thermal increase was monitored at this time. Nevertheless, it seems that the burn was due to mechanical stress applied on a weak solder because the copper cylinder was massive. The burn spread radially from one turn to the next one on about fifteen turns. To fix the coil, thirty turns were removed from the winding. The new external diameter is $108 \mathrm{~mm}$. In this configuration, the outer diameter is smaller than the maximal outer diameter of the final prototype.

Once the coil is fixed and soldered again, it will be tested under the previously explained conditions. 
(a)

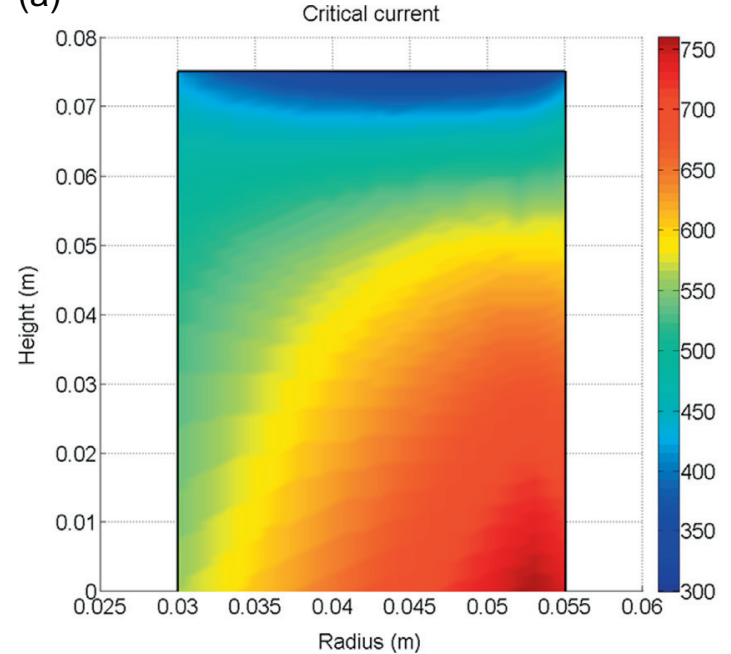

(b)

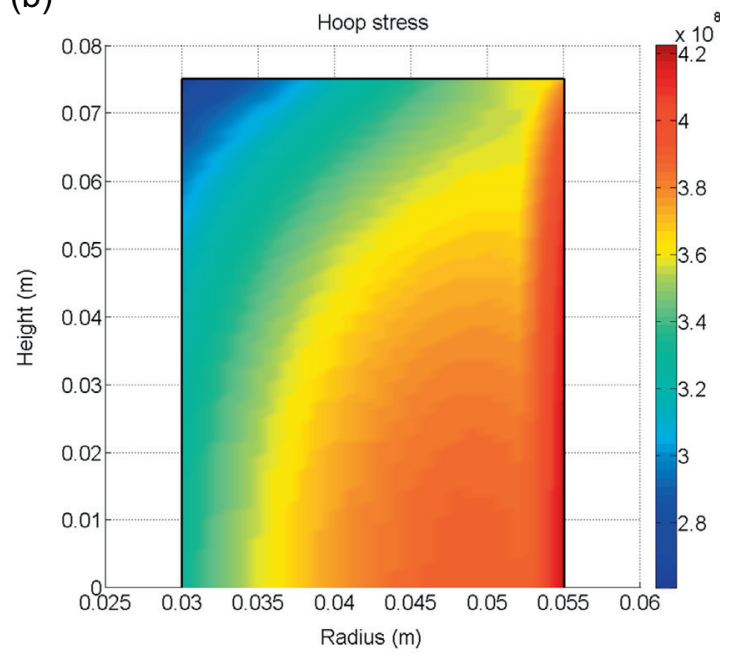

Fig. 8. (a) Critical current distribution on the cross section of the final prototype according to the magnetic field orientation and intensity. (b) Mechanical stress distribution on the cross section of the final prototype in the nominal working state.

Table 3. Double pancake information.

\begin{tabular}{lc}
\hline Characteristics & \\
\hline Inner diameter & $54 \mathrm{~mm}$ \\
Outer diameter & $120 \mathrm{~mm}$ \\
Top pancake & $240 \mathrm{turns}$ \\
Bottom pancake & $234 \mathrm{turns}$ \\
Measured inductance & $11.14 \mathrm{mH}$ \\
Self-field in the center & $2 \mathrm{~T}$ \\
\hline
\end{tabular}

(a)

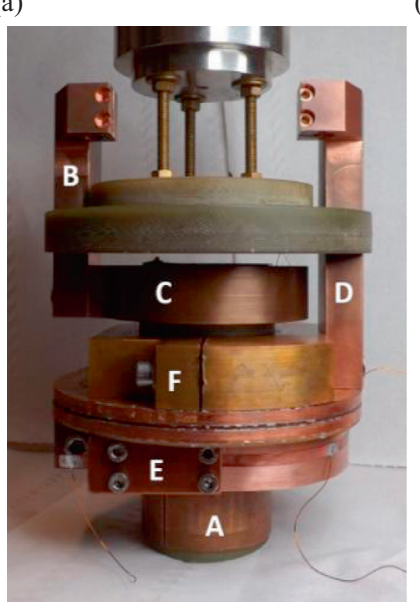

(b)

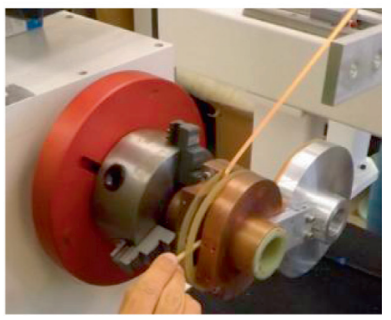

Fig. 9. (a) Double pancake assembly before wrapping. (b) Winding of the first pancake while the other half of the tape is wound on a satellite.

Nevertheless, the experiment protocol will be modified. The voltage signal of the coil was noisy especially because of the sample coil inductance. In order to improve the voltage signal measurement, a constant current, lower than the expected critical current at $4.2 \mathrm{~K}$, will be passed through the sample coil and the helium temperature will be increased [29] until the coil quenches. Hence, the voltage due to the coil inductance during a current ramp will be suppressed.

\section{Conclusion and further work}

This work is part of the global research about superconductivity. HTS applications are still complex and magnet insert fabrication challenging.

In this configuration, the winding is subject to severe mechanical stresses. A non-negligible transverse component of the field is applied on the edges of the windings. This considerably decreases the tape performance in these areas. It is responsible for a lower limiting critical current of the final prototype. The geometry of the final prototype, especially the edges of the winding, has to be optimized in order to reduce as much as possible the maximal value of the transverse component on the winding.

The calculated current margin is below $60 \%$ without any optimization of the final prototype geometry. Nevertheless, this limiting critical current calculation lacks information as it only takes the tape performance into consideration.

Eventually, the first wound prototype has been tested under $16 \mathrm{~T}$ up to $200 \mathrm{~A}$. It has been damaged and fixed. One of the wound tape extremities burnt so the copper pieces were reduced and the burnt part cut so the pancake can still reach the current leads. The outer diameter is now reduced to $108 \mathrm{~mm}$. Experiments will soon continue with test conditions as close as possible to that of the HTS insert.

This work was possible, thanks to our colleagues J. Vialle (winding process), C. Mollard (mechanics), G. Novitchi (chemistry) and Y. Miyoshi (design, fabrication and set up of the probes). It is founded by ANR, project NOUGAT - NOUvelle 
Génération d'Aimant supraconducteur pour la production de Teslas avec une consommation électrique réduite, and supported by the LNCMI-CNRS, member of EMFL.

\section{References}

1. H. Maeda, Y. Yanagisawa, IEEE Trans. Appl. Supercond. 23, 4602412 (2014)

2. "A Comparison of Superconductor Critical Currents" (NHMFL, 2014), available on https://nationalmaglab. org/magnet-development/applied-superconductivitycenter/plots

3. Data available on https://www.bruker.com/fr/ products $/ \mathrm{mr} / \mathrm{nmr} / \mathrm{magnets} / \mathrm{magnets} /$ avance-1000/ overview . $\mathrm{html}$ ? amp $\% 3 \mathrm{BL}=2$

4. W.D. Markiewicz et al., IEEE Trans. Appl. Supercond. 22, $4300704(2012)$

5. S. Awaji et al., IEEE Trans. Appl. Supercond. 24 (2014)

6. Q. Wang et al., IEEE Trans. Appl. Supercond. 23, 4300905 (2013)

7. J. Bascuñán et al., IEEE Trans. Appl. Supercond. 24, 6400205 (2014)

8. F. Borgnolutti et al., IEEE Trans. Appl. Supercond. 26 (2016)

9. J.X. Jin, S.X. Dou, C. Grantham, D. Sutanto, Towards electrical applications of high $T_{c}$ superconductors, in Fourth International Conference on Advances in Power System Control, Operation and Management, 1997, p. $427-432$

10. Available on http://www. superpower-inc.com/content/ $2 \mathrm{~g}$-hts-wire
11. D. Uglietti, H. Kitaguchi, S. Choi, T. Kiyoshi, IEEE Trans. Appl. Supercond. 19 (2009)

12. P.M. Leys et al., IEEE Trans. Appl. Supercond. 23, 8000604 (2013)

13. T.S. Lee et al., Supercond. Sci. Technol. 27 (2014)

14. S.B. Kim et al., IEEE Trans. Appl. Supercond. 23 (2013)

15. S. Hahn, D.K. Park, J. Bascuñán, Y. Iwasa, IEEE Trans. Appl. Supercond. 21 (2011)

16. W. Markiewicz, IEEE Trans. Appl. Supercond. 18 (2008)

17. Y. Yanagisawa et al., Physica C 495, 15 (2013)

18. http://www. cedrat.com/en/software/flux.html

19. Y. Miyoshi, X. Chaud, F. Drebray, B. Vincent, P. Tixador, J.-M. Rey, H. Oguro, S. Awaji, K. Watanabe, G. Nishijima, H. Kitaguchi, IEEE Trans. Appl. Supercond. 23 (2013)

20. Y. Miyoshi et al., Physica C 516, 31 (2015)

21. H.W. Weijers, U.P. Trociewitz, W.D. Markiewicz, J. Jiang, D. Myers, E.E. Hellstrom, A. Xu, J. Jaroszynski, P. Noyes, Y. Viouchkov, D.C. Larbalestier, IEEE Trans. Appl. Supercond. 20 (2010)

22. V. Braccini et al., Supercond. Sci. Technol. 24 (2011)

23. M. Solovyov et al., Supercond. Sci. Technol. 26 (2013)

24. G. Nishijima, Y. Tsuchiya, H. Kitaguchi, T. Nishimura, T. Kato, IEEE Trans. Appl. Supercond. 23 (2013)

25. Y.B. Kim, C.F. Hempstead, A.R. Strnad, Phys. Rev. Lett. 9, 306 (1962)

26. Z. Jiang et al., IEEE Trans. Appl. Supercond. 16 (2006)

27. A. Otsuka, T. Kioshi, IEEE Trans. Appl. Supercond. 18 (2008)

28. T. Benkel et al., IEEE Trans. Appl. Supercond. 27 (2017)

29. R. Gupta et al., IEEE Trans. Appl. Supercond. 26 (2016) 